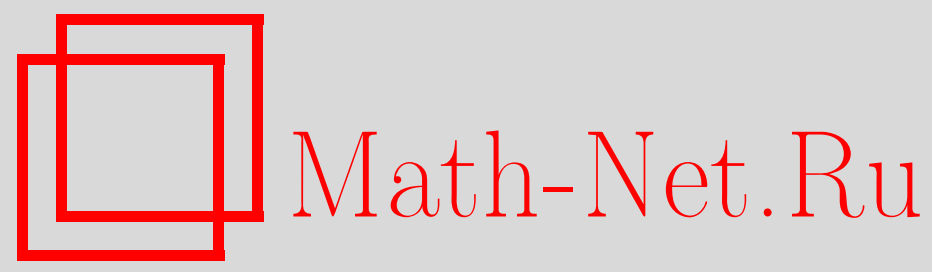

А. М. Гайсин, Максимальный член измененного ряда Дирихле, Матем. заметки, 2001, том 69, выпуск 6, 828-842

DOI: https://doi.org/10.4213/mzm697

Использование Общероссийского математического портала Math-Net.Ru подразумевает, что вы прочитали и согласны с пользовательским соглашением http://www . mathnet.ru/rus/agreement

Параметры загрузки:

IP: 52.90 .164 .192

26 апреля 2023 г., 18:25:07

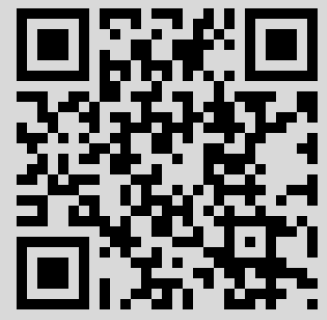


УДК 517.53

\section{МАКСИМАЛЬНЫЙ ЧЛЕН ИЗМЕНЕННОГО РЯДА ДИРИХЛЕ}

\section{А. М. Гайсин}

Найден критерий того, чтобы логарифм максимального члена ряда Дирихле, абсолютно сходящегося в некоторой полуплоскости, на асимптотическом множестве был эквивалентен логарифму максимального члена его адамаровской композиции с любым другим рядом Дирихле из определенного класса.

Библиография: 8 названий.

Пусть $\Lambda=\left\{\lambda_{n}\right\} \quad\left(0<\lambda_{n} \uparrow \infty\right)$ - последовательность, удовлетворяющая условию

$$
\varlimsup_{n \rightarrow \infty} \frac{\ln n}{\ln \lambda_{n}}=a<\infty .
$$

Через $D_{0}(\Lambda)$ обозначим класс всех функций $F(s)$, представимых в полуплоскости $\Pi_{0}=$ $\{s: \operatorname{Re} s<0\}$ рядами Дирихле

$$
F(s)=\sum_{n=1}^{\infty} a_{n} e^{\lambda_{n} s} \quad(s=\sigma+i t)
$$

сходящимися лишш в данной полуплоскости. Из условия (1) следует, что

$$
\varlimsup_{n \rightarrow \infty} \frac{\ln n}{\lambda_{n}}=0 .
$$

Так что ряд (2) сходится в полуплоскости П 0 абсолютно и равномерно, а его сумма $F(s)-$ аналитическая в $\Pi_{0}$ функция [1].

Наряду с рядом (2) введем в рассмотрение измененньй ряд Дирихле

$$
F^{*}(s)=\sum_{n=1}^{\infty} a_{n} b_{n} e^{\lambda_{n} s},
$$

где последовательность $\left\{b_{n}\right\}\left(b_{n} \neq 0\right.$ при $\left.n \geqslant N\right)$ удовлетворяет условию

$$
\varlimsup_{n \rightarrow \infty} \frac{\ln \left|b_{n}\right|}{\lambda_{n}}=0 .
$$

Работа выполнена при финансовой поддержке Российского фонда фундаментальных исследований, грант № 99-01-00655. 
Поскольку $F \in D_{0}(\Lambda)$, то [1]

$$
\varlimsup_{n \rightarrow \infty} \frac{\ln \left|a_{n}\right|}{\lambda_{n}}=0 .
$$

Следовательно, с учетом (4) заключаем, что ряд (3) также сходится в полуплоскости $\Pi_{0}$, причем абсолютно и равномерно, а его сумма $F^{*}(s)$ является аналитической в данной полуплоскости.

Заметим, что при условии (4) имеет смысл рассматривать также абсолютно сходящиеся в полуплоскости П

$$
\sum_{n=N}^{\infty} a_{n} b_{n}^{-1} e^{\lambda_{n} s}
$$

Пусть $\mu(\sigma)$ и $\mu^{*}(\sigma)$ - максимальные члены рядов $(2)$ и (3) соответственно, т.е.

$$
\mu(\sigma)=\max _{n \geqslant 1}\left\{\left|a_{n}\right| e^{\lambda_{n} \sigma}\right\}, \quad \mu^{*}(\sigma)=\max _{n \geqslant 1}\left\{\left|a_{n} b_{n}\right| e^{\lambda_{n} \sigma}\right\} .
$$

Через $\nu=\nu(\sigma)$ и $k=k(\sigma)$ в дальнейшем будем обозначать центральные индексы рядов $(2)$ и $(3)$, т.е.

$$
\nu(\sigma)=\max \left\{n \geqslant 1:\left|a_{n}\right| e^{\lambda_{n} \sigma}=\mu(\sigma)\right\}, \quad k(\sigma)=\max \left\{n \geqslant 1:\left|a_{n} b_{n}\right| e^{\lambda_{n} \sigma}=\mu^{*}(\sigma)\right\} .
$$

В статье исследуется вопрос: при каких условиях на последовательность $\left\{b_{n}\right\}$ при $\sigma \rightarrow 0-$ вдоль достаточно массивного множества $A \subset[-1,0)$ имеет место следующее асимптотическое равенство

$$
\ln \mu(\sigma)=(1+o(1)) \ln \mu^{*}(\sigma) .
$$

Напомним, что множество $A$ назьвается асимптотическим, а его дополнение $e=$ $[-1,0) \backslash A-$ исключительным.

Пусть $e-$ измеримое по мере Лебега $m$ подмножество полуинтервала $[-1,0)$. Bepxней и нижней плотностями множества $e$ (обозначаемьми $D e$ и $d e$ соответственно) назьваются величины

$$
D e=\varlimsup_{\sigma \rightarrow 0-} \frac{m(e \cap[\sigma, 0))}{|\sigma|}, \quad d e=\varliminf_{\sigma \rightarrow 0}-\frac{m(e \cap[\sigma, 0))}{|\sigma|} .
$$

В дальнейшем подразумевается, что все исключительные множества представляют собой объединение счетного числа отрезков вида

$$
\left[a_{n}, a_{n}^{\prime}\right] \quad\left(-1 \leqslant a_{1}<a_{1}^{\prime} \leqslant a_{2}<a_{2}^{\prime} \leqslant \cdots \leqslant a_{n}<a_{n}^{\prime} \leqslant \cdots<0\right) .
$$

Пусть $L$ - класс положительных, непрерывных и неограниченно возрастающих на $[0, \infty)$ функций. Через $W$ обозначим подкласс функций $w=w(x)$ из $L$ таких, что

$$
\int_{1}^{\infty} \frac{w(x)}{x^{2}} d x<\infty .
$$

Пусть $D(\Lambda)$ - класс всех целых функций $F(s)$, представимых абсолютно сходящимися во всей плоскости рядами Дирихле (2). Если

$$
\varlimsup_{n \rightarrow \infty} \frac{1}{\lambda_{n}} \ln \left(\left|b_{n}\right|+\frac{1}{\left|b_{n}\right|}\right)<\infty,
$$

то ряд (3) также абсолютно сходится во всей плоскости, $F^{*} \in D(\Lambda)$.

В работе [2] установлена следующая 
Теорема А. Пусть $\left\{b_{n}\right\}$ - некоторая последовательность комплексных чисел, удовлетворяющая условию (5).

Для того чтобь для любой функиии $F \in D(\Lambda)$ при $\sigma \rightarrow+\infty$ вне некоторого множества е $\subset[0, \infty)$ отрезков $\left[c_{n}, c_{n}^{\prime}\right]\left(0 \leqslant c_{1}<c_{1}^{\prime} \leqslant c_{2}<c_{2}^{\prime} \leqslant \cdots \leqslant c_{n}<c_{n}^{\prime} \leqslant \ldots\right)$ суммарной конечной длины имело место асимптотическое равенство

$$
\ln \mu(\sigma)=(1+o(1)) \ln \mu^{*}(\sigma),
$$

необходимо и достаточно, чтобь для некоторой функиии $w \in W$ выполнялись оценки

$$
\left|b_{n}\right|+\frac{1}{\left|b_{n}\right|} \leqslant e^{w\left(\lambda_{n}\right)} \quad(n \geqslant N) .
$$

Цель статьи - перенести этот результат на ряды Дирихле, сходяшиеся лишь в полуплоскости $\Pi_{0}$. В этой ситуации возникают серьезные трудности, связанные, в первую очередь, с оценкой размеров исключительных множеств $\{e\} \subset[-1,0)$ при $\sigma \rightarrow 0-$. По этой причине обычно приходится требовать выполнения некоторой оценки снизу для максимального члена ряда (2) (см. например, в [3]-[5]). Кроме того, в дальнейшем мы будем рассматривать только те последовательности $\left\{b_{n}\right\}$, которые, по крайней мере, удовлетворяют условию (6). Это вызвано тем, что в нашей ситуации теоремы типа Бореля-Неванлинны содержательны лишь для мажорант $w$ из класса $W$ (см. [5, лемма 5]).

Пусть $N$ - класс функций $\Phi$, принадлежащих $L$ и таких, что

1) $(\Phi(x) / x) \uparrow \infty$ при $x \rightarrow \infty$;

2) $x \Phi(x)<\Phi(k x)$ при $x \geqslant x_{0}$, где $k$ - некоторая постоянная.

Отметим, что класс $N$ содержит сужения на полуось $[0, \infty)$ всех так называемых $N$ функций, удовлетворяюших $\Delta_{3}$-условию [6]. Примерами функций из $N$, в частности, являются $e^{x}, e^{x^{2}}, e^{e^{x}}, x^{\ln x}$ и т.д. Все эти функции растут быстрее любой степени $x^{n}$. Это не случайно, так как в силу условия 2 ) при $x \geqslant x_{0} k^{n}$ выполнены неравенства [6]

$$
\Phi(x)>\frac{x}{k} \Phi\left(\frac{x}{k}\right)>\frac{x^{2}}{k^{3}} \Phi\left(\frac{x}{k^{2}}\right)>\cdots>\frac{x^{n}}{k^{n(n+1) / 2}} \Phi\left(\frac{x}{k^{n}}\right)>\frac{\Phi\left(x_{0}\right)}{k^{n(n+1) / 2}} x^{n} .
$$

Каждой функции $\Phi$ из $N$ поставим в соответствие ее обратную функцию $\varphi$. Тогда получим новьй класс функций, обозначим его через $N^{-1}$. Таким образом, классы $N=\{\Phi\}$ и $N^{-1}=\{\varphi\}$ состоят из взаимно обратных функций. Заметим (это следует из условия 1$))$, что если $\varphi \in N^{-1}$, то $(\varphi(t) / t) \downarrow 0$ при $t \uparrow \infty$.

Пусть $\varphi$ - некоторая функция из $L$; обозначим

$$
W_{\varphi}=\{w: w \in W, \varphi(t) J(t ; w)=o(1), t \rightarrow \infty\},
$$

где

$$
J(t ; w)=\int_{t}^{\infty} \frac{w(x)}{x^{2}} d x .
$$

И, наконец, пусть $\Phi \in N$,

$$
\bar{D}_{0}(\Lambda, \Phi)=\left\{F \in D_{0}(\Lambda): \sup _{\tau>0} \varlimsup_{\sigma \rightarrow 0-} \frac{\ln \mu_{F}(\sigma)}{|\sigma| \Phi(\tau /|\sigma|)}>0\right\},
$$

где $\mu_{F}(\sigma)$ - максимальный член ряда (2). В дальнейшем максимальньй член этого ряда будем обозначать, как и прежде, через $\mu(\sigma)$.

Сформулируем теперь основной результат. 
Теорема В. Пусть $\Phi$ - некоторая фиксированная функиия из класса $N$.

Для того чтобы для любой функиии $F \in \bar{D}_{0}(\Lambda, \Phi)$ при $\sigma \rightarrow 0-$ вне некоторого исключительного множества е $\subset[-1,0)$ нулевой нижней плотности де имело место асимптотическое равенство

$$
\ln \mu(\sigma)=(1+o(1)) \ln \mu^{*}(\sigma)
$$

необходимо и достаточно, чтобы для некоторой функиии $w \in W_{\varphi}$ выполнялись оценки

$$
\left|b_{n}\right|+\frac{1}{\left|b_{n}\right|} \leqslant e^{w\left(\lambda_{n}\right)} \quad(n \geqslant N) .
$$

Здесъ $\varphi-$ функиия, обратная $к \Phi$.

Приведем следствие, вытекающее из теоремы В.

Пусть область сходимости степенного ряда

$$
f(z)=\sum_{n=0}^{\infty} a_{n} z^{n}
$$

- единичный круг $D=\{z:|z|<1\}$. Сделаем замену $z=e^{s}$. Тогда $F \in D_{0}(\Lambda)$, где $\Lambda=\{0,1,2, \ldots, n, \ldots\}$, a $F(s)=f\left(e^{s}\right)(s=\sigma+i t)$.

Пусть $\Phi \in N$, а $\varphi$ - функция, обратная к $\Phi$. Поскольку $e^{\sigma}=|z|=r \rightarrow 1-$, $|\sigma|=|\ln r|=1-r+o(1-r)$ при $\sigma \rightarrow 0-$, а $\varphi(2 t) \leqslant 2 \varphi(t)$, то классу $\bar{D}_{0}(\Lambda, \Phi)$ функций $F$ будет соответствовать следуюший класс:

$$
\bar{K}(\Phi)=\left\{f: \sup _{\tau>0} \varlimsup_{r \rightarrow 1-} \frac{\ln \mu_{f}(r)}{(1-r) \Phi(\tau /(1-r))}>0\right\},
$$

где $f$ - функция, заданная в круге $D$ рядом $(10)$, a $\mu_{f}(r)$ - максимальньй член этого ряда. Через $\mu_{f}^{*}(r)$ обозначим максимальный член измененного степенного ряда

$$
f^{*}(z)=\sum_{n=0}^{\infty} a_{n} b_{n} z^{n}
$$

где $\left\{b_{n}\right\} \quad\left(b_{n} \neq 0\right.$ при $\left.n \geqslant N\right)$ - любая последовательность комплексных чисел, удовлетворяющая условию (6).

Если $e_{1} \subset[0,1)$ - образ множества $e \subset[0,1)$ при отображении $r=e^{\sigma}$, то условие $d e=0$ означает, что $d^{\prime} e_{1}=0$, где

$$
d^{\prime} e_{1}=\varliminf_{r \rightarrow 1-} \frac{m\left(e_{1} \cap[r, 1)\right)}{1-r} .
$$


Теорема С. Пусть $\Phi$ - некоторая фиксированная функиия из класса $N$. Для того чтобы для любой функции $f \in \bar{K}(\Phi)$ при $r \rightarrow 1-$ вне некоторого исключительного множества е $e_{1} \subset[0,1), d^{\prime} e_{1}=0$, выполнялось асимптотическое равенство

$$
\ln \mu_{f}(r)=(1+o(1)) \ln \mu_{f}^{*}(r),
$$

необходимо и достаточно, чтобы для некоторой функции $w \in W_{\varphi}$ выполнялись оченки (9). Здесъ $\varphi-$ функиия, обратная $к \Phi$.

1. Вспомогательные результаты. Пусть $L$ - класс функций, введенньй вьше, а $H$ - подкласс $L$, состоящий из функций $\Phi$, для которых

a) $\varphi(2 t) \leqslant a \varphi(t) \quad(0<a<\infty)$;

в) $\varphi(t) t^{-1} \ln t=o(1), t \rightarrow \infty$,

где $\varphi$ - функция, обратная к $\Phi$. Ясно, что $N \subset H$. Действительно, если $\Phi \in N$, то $\varphi\left(t_{1}\right) / t_{1}>\varphi\left(t_{2}\right) / t_{2}$ при $t_{1}<t_{2}$. Следовательно, $\varphi(2 t)<2 \varphi(t)$. Условие в) немедленно следует из оценки (7) (если положить $n=2$, видно, что $\varphi(t)=O\left(t^{1 / 2}\right)$ при $t \rightarrow \infty$ ).

Лемма 1. Пусть $u(t)$ - непрерывная, неубывающая на $[-1,0)$ функиия, $u(t) \rightarrow \infty$ при $t \rightarrow 0-$, причем для некоторой функиии $\Phi$ из Н выполняется условие

$$
\varlimsup_{t \rightarrow 0-} \frac{e^{u(t)}}{\Phi(1 /|t|)}>0 .
$$

Если для некоторой функиии $w$ из $L$ имеет место асимптотика

$$
\frac{\varphi(x) w(x)}{x}=o(1), \quad x \rightarrow \infty
$$

$(\varphi-$ функиия, обратная $\kappa \Phi), a v=v(t)-$ решение уравнения

$$
w(v)=e^{u(t)},
$$

то при $t \rightarrow 0-$ вне некоторого множества е $e_{1} \subset[-1,0)$ нулевой нижней плотности dе 1 имеет место асимптотика

$$
\frac{w(v(t))}{v(t)|t|}=o(1)
$$

Смысл условия (13) в том, что при $t \rightarrow 0-$ вне исключительного множества $e_{1}$ выполняется неравенство

$$
t+\frac{w(v(t))}{v(t)}=t(1+o(1))<0 .
$$

Лемма 2. Пусть выполняется условие (11). Если $\varphi-$ функиия, обратная $к \Phi$, a $w \in W_{\varphi}$, то при $t \rightarrow 0-$ вне некоторого множества $e_{2} \subset[-1,0)$, de $_{2}=0$, имеет место асимптотическое равенство

$$
u(t+h(t))=u(t)+o(1),
$$

где $h(t)=w(v(t)) / v(t)$, a $v=v(t)-$ решение уравнения (12).

Леммы 1 и 2 установлены в работе [5]. 
ЗАМЕчАниЕ. В [5] показано, что множество $e=e_{1} \cup e_{2}$ имеет также нулевую нижнюю плотность, т.е. $d e=0$.

Лемма 3. Пусть $\alpha=\alpha(t)$ - положительная, неубьвающая и непрерывная справа на луче $[a, \infty)(0<a<\infty)$ функиия, причем

$$
\int_{a}^{\infty} \frac{\alpha(x)}{x^{2}} d x<\infty
$$

$E с л и \varphi \in L, \varphi(t) / t \downarrow n p u t \uparrow u$

$$
\varlimsup_{t \rightarrow \infty} \varphi(t) J(t ; \alpha)>0, \quad J(t ; \alpha)=\int_{t}^{\infty} \frac{\alpha(x)}{x^{2}} d x,
$$

то существует подпоследовательность $\left\{t_{n_{j}}\right\}$ последовательности $\left\{t_{n}\right\}$ всех точек разрыва функиии $\alpha(t)$ такая, что

$$
\varphi\left(t_{n_{j}}\right) J\left(t_{n_{j}} ; \alpha\right) \geqslant d>0 \quad(j \geqslant 1) .
$$

ДоКАЗАТЕЛЬСТво. Из (14) получаем, что для некоторой последовательности $\left\{\tau_{j}\right\}$ $\left(a<\tau_{j} \uparrow \infty\right)$ имеет место оценка

$$
\varphi\left(\tau_{j}\right) J\left(\tau_{j} ; \alpha\right) \geqslant q>0 \quad(j \geqslant 1) .
$$

Если $\tau_{j} \in\left[t_{n-1}, t_{n}\right)$, то

$$
\begin{aligned}
\varphi\left(\tau_{j}\right) J\left(\tau_{j} ; \alpha\right) & =\varphi\left(\tau_{j}\right) \int_{\tau_{j}}^{t_{n}} \frac{\alpha(x)}{x^{2}} d x+\varphi\left(\tau_{j}\right) J\left(t_{n} ; \alpha\right) \\
& \leqslant \frac{\varphi\left(\tau_{j}\right)}{\tau_{j}} \alpha\left(t_{n-1}\right)+\varphi\left(t_{n}\right) J\left(t_{n} ; \alpha\right) .
\end{aligned}
$$

Поскольку $t_{n-1} \leqslant \tau_{j}<t_{n}$, a

$$
\frac{\varphi\left(\tau_{j}\right)}{\tau_{j}} \leqslant \frac{\varphi\left(t_{n-1}\right)}{t_{n-1}}
$$

то из (16) получаем, что

$$
\begin{aligned}
\varphi\left(\tau_{j}\right) J\left(\tau_{j} ; \alpha\right) & \leqslant \frac{\varphi\left(t_{n-1}\right)}{t_{n-1}} \alpha\left(t_{n-1}\right)+\varphi\left(t_{n}\right) J\left(t_{n} ; \alpha\right) \\
& \leqslant \varphi\left(t_{n-1}\right) J\left(t_{n-1} ; \alpha\right)+\varphi\left(t_{n}\right) J\left(t_{n} ; \alpha\right)=J_{n-1}+J_{n} .
\end{aligned}
$$

Если $\max \left(J_{n-1}, J_{n}\right)=J_{n_{j}}\left(n_{j}=n-1\right.$ или $\left.n_{j}=n\right)$, то

$$
\frac{1}{2} \varphi\left(\tau_{j}\right) J\left(\tau_{j} ; \alpha\right) \leqslant J_{n_{j}}=\varphi\left(t_{n_{j}}\right) J\left(t_{n_{j}} ; \alpha\right) .
$$

Отсюда с учетом (15) получаем требуемую оценку. 
Теперь вкратце остановимся на некоторых свойствах максимального члена ряда Дирихле. Хорошо известно геометрическое описание максимального члена степенного ряда или ряда Дирихле, задающего целую функцию, через выпуклый полигон Ньютона (см., например, [1]). Аналогичное описание максимального члена степенного ряда, сходящегося лишш в единичном круге, дается также в ряде работ (см., например, в [7]).

Построим выпукльй полигон Ньютона для ряда Дирихле (2), абсолютно сходящегося лишь в полуплоскости П 0 . Для этого, предполагая, что $\sup _{n}\left|a_{n}\right|=\infty$ (можно также считать, что $\left.a_{1} \neq 0\right)$, отметим на плоскости $X o Y$ точки $P_{n}=\left(\lambda_{n}, g_{n}\right)$, где $g_{n}=-\ln \left|a_{n}\right|$ (если $a_{n}=0$, то полагаем $g_{n}=\infty$ ). Поскольку $F \in D_{0}(\Lambda)$, то

$$
\varlimsup_{n \rightarrow \infty} \frac{\ln \left|a_{n}\right|}{\lambda_{n}}=0 .
$$

Учитьвая это, через $Q(F)$ обозначим выпуклую оболочку точек $P_{n}(n \geqslant 1)$. Пусть $\gamma(x)=\inf \{y:(x, y) \in Q(F)\}$. Линия, описьваемая уравнением $y=\gamma(x)(x \geqslant 1)$, назьвается диаграммой или выпуклым полигоном Ньютона [7]. Из (17) следует, что диаграмма Ньютона, обозначим ее $L(F)$, есть выпуклая вниз ломаная линия.

Пусть $F \in D_{0}(\Lambda)$,

$$
F(s)=\sum_{n=1}^{\infty} a_{n} e^{\lambda_{n} s}, \quad \sup \left|a_{n}\right|=\infty
$$

Положим

$$
\widehat{F}(s)=\sum_{n-1}^{\infty} T_{n} e^{\lambda_{n} s}, \quad T_{n}=e^{-\gamma\left(\lambda_{n}\right)} \quad(n \geqslant 1) .
$$

Функция $\widehat{F}$ называется мажсорантой Ньютона функции $F \in D_{0}(\Lambda)$.

Пусть $\gamma\left(\lambda_{n}\right)=G_{n}(n \geqslant 1)$. Тогда $\left(\lambda_{n}, G_{n}\right) \in L(F)$. Для бесконечного множества значений $\lambda_{n}$, в частности, для абсцисс $\lambda_{n_{i}}\left(i \geqslant 1, n_{1}=1\right)$ всех вершин полигона $L(F)$ имеем $G_{n}=-\ln \left|a_{n}\right|$. Отметим, что точка $P_{n}=\left(\lambda_{n},-\ln \left|a_{n}\right|\right)$ лежит либо на полигоне $L(F)$ (точка $P_{n_{i}}$ обязательно лежит на полигоне), либо над ним. Угловой коэффициент отрезка, соединяющего вершины $P_{n_{i}}$ и $P_{n_{i+1}}$ полигона $L(F)$, равен

$$
R_{i}=\frac{G_{n_{i+1}}-G_{n_{i}}}{\lambda_{n_{i+1}}-\lambda_{n_{i}}} \quad\left(i \geqslant 1, \quad \lambda_{1}=1\right) .
$$

Ясно, что $R_{i} \uparrow 0$ при $i \rightarrow \infty$. Следовательно, при $R_{i-1} \leqslant \sigma<R_{i}$ центральный индекс $\nu(\sigma)=n_{i}=$ const, a $\ln \mu(\sigma)=\ln \left|a_{n_{i}}\right|+\lambda_{n_{i}} \sigma$ [1]. Отсюда, в частности, следует, что $\mu(\sigma)=\hat{\mu}(\sigma), \nu(\sigma)=\hat{\nu}(\sigma)$, где $\hat{\mu}(\sigma)$ и $\hat{\nu}(\sigma)$ - максимальньй член и центральньй индекс ряда (18). Известно также, что функция $\ln \mu(\sigma)$ непрерывна, а при $\sup _{n}\left|a_{n}\right|=\infty$ неограниченно возрастает на интервале $[-1,0)[1]$.

2. Доказательство теоремы В. Достаточность. Пусть $F \in \bar{D}_{0}(\Lambda, \Phi)$, где $\Phi \in N$, а $\varphi\left(\varphi \in N^{-1}\right)$ - обратная к $\Phi$ функция.

Последовательность $\left\{b_{n}\right\}$ подчинена условию: существует $w \in W_{\varphi}$ такая, что

$$
\left|b_{n}\right|+\frac{1}{\left|b_{n}\right|} \leqslant e^{w\left(\lambda_{n}\right)} \quad(n \geqslant N) .
$$


Следовательно, имеется функция $w^{*} \in W_{\varphi}$ такая, что $w(x)=o\left(w^{*}(x)\right)$ и $\sqrt{x} \leqslant w^{*}(x)$ при $x \rightarrow \infty[5]$.

Пусть $v=v(\sigma)$ - решение уравнения

$$
w^{*}(v)=3 \ln \mu(\sigma)
$$

Положим

$$
R_{v}=\sum_{\lambda_{j}>v}\left|a_{j}\right| e^{\lambda_{j} \sigma}, \quad h=\frac{w^{*}(v)}{v}, \quad v=v(\sigma) .
$$

Пусть $m \geqslant 1$ такое, что

$$
\sum_{n=1}^{\infty} \frac{1}{\lambda_{n}^{m}}<\infty
$$

В силу условия (1) такое $m$ существует.

Поскольку $F \in \bar{D}_{0}(\Lambda, \Phi)$, то существует последовательность $\left\{\tau_{j}\right\}\left(0<\tau_{j} \uparrow 0\right)$ такая, что при некоторых $\tau>0, P>0$ и $\sigma=\tau_{j}(j \geqslant 1)$ выполнена оценка

$$
\frac{\ln \mu(\sigma)}{|\sigma| \Phi(\tau /|\sigma|)} \geqslant P>0
$$

Пользуясь тем, что $\Phi \in N$, отсюда при некотором $q(0<q<1)$ получаем, что

$$
\ln \mu(\sigma) \geqslant \frac{\Phi\left(\tau|\sigma|^{-1}\right)}{|\sigma|^{-1}}>P \tau \Phi_{1}\left(|\sigma|^{-1}\right), \quad \sigma=\tau_{j} \quad\left(j \geqslant j_{0}\right),
$$

где $\Phi_{1}(t)=\Phi(q t)$. Легко проверяется, что $\Phi_{1} \in N$. Значит, $\Phi_{1} \in H$. Следовательно, применяя леммы 1 и 2 , а также учитывая замечание к ним, при $\sigma \rightarrow 0-$ вне некоторого множества $e_{1} \subset[-1,0)$ нулевой нижней плотности получаем, что

$$
\begin{aligned}
& \sigma+h=\sigma\left(1+\frac{h}{\sigma}\right)=\sigma(1+o(1))<0, \\
& \mu(\sigma+h)<\mu(\sigma)^{1+o(1)}, \quad h=\frac{w^{*}(v)}{v},
\end{aligned}
$$

где $v=v(\sigma)$-решение уравнения (19). Если $\left\{\tau_{j}\right\}$-последовательность, фигурируюшая в оценках (20), то (это легко усматривается из доказательств лемм 1 и 2) [5]

$$
\frac{m\left(e_{1} \cap\left[\tau_{j}, 0\right)\right)}{\left|\tau_{j}\right|}=o(1), \quad \tau_{j} \rightarrow 0-.
$$

Далее, при $\sigma \in\left[\sigma_{0}, 0\right) \backslash e_{1}$ имеем

$$
R_{v} \leqslant \mu(\sigma+h) \sum_{\lambda_{n}>v} e^{-h \lambda_{n}} \leqslant\left(\sum_{n=1}^{\infty} \frac{1}{\lambda_{n}^{m}}\right) \mu(\sigma+h) \exp \left[\max _{t \geqslant v} \psi(t)\right]
$$


где $\psi(t)=m \ln t-h t$. Поскольку $\psi^{\prime}(t)=0$ в точке $t=t_{0}$,

$$
t_{0}=\frac{m}{h}=m \frac{v}{w^{*}(v)} \leqslant m \sqrt{v}<v=v(\sigma)
$$

при $\sigma_{1} \leqslant \sigma<0$, то

$$
R_{v} \leqslant C \mu(\sigma+h) \exp [-v(1+o(1)) h], \quad C=\sum_{n=1}^{\infty} \frac{1}{\lambda_{n}^{m}}
$$

Отсюда, учитывая (21), получаем, что при $\sigma \rightarrow 0-$ вне исключительного множества $e_{1} \subset[-1,0)$, для которого $d e_{1}=0$, выполнена оценка

$$
R_{v} \leqslant C \mu(\sigma)^{1+o(1)} \exp \left[-w^{*}(v)(1+o(1))\right]=\mu(\sigma)^{-2(1+o(1))} .
$$

Значит, при $\sigma_{2} \leqslant \sigma<0, \sigma \notin e_{1}$, имеем $\lambda_{\nu(\sigma)} \leqslant v(\sigma)$, где $\nu=\nu(\sigma)$ - центральньй индекс ряда (2). Тогда при $\sigma \rightarrow 0-$ вне $e_{1}$

$$
\mu(\sigma)=\left|a_{\nu}\right| e^{\lambda_{\nu} \sigma}=\left|a_{\nu} b_{\nu}\right| e^{\lambda_{\nu} \sigma}\left|b_{\nu}\right|^{-1} \leqslant \mu^{*}(\sigma) e^{w(v)}=\mu^{*}(\sigma) \mu(\sigma)^{o(1)} .
$$

Следовательно, при $\sigma \rightarrow 0-$ вне некоторого множества $e_{1} \subset[-1,0)$ нулевой нижней плотности

$$
(1+o(1)) \ln \mu(\sigma) \leqslant \ln \mu^{*}(\sigma) .
$$

Далее, поскольку $\left|b_{n}\right| \leqslant e^{w\left(\lambda_{n}\right)}(n \geqslant N)$, то при $\sigma_{2} \leqslant \sigma<0$

$$
\mu^{*}(\sigma)=\left|a_{k} b_{k}\right| e^{\lambda_{k} \sigma} \leqslant \mu(\sigma) e^{w\left(\lambda_{k}\right)}
$$

где $k=k(\sigma)$ - центральный индекс ряда (3).

Пусть $x=x(\sigma)$ - решение уравнения $w^{*}(x)=3 \ln \mu^{*}(\sigma)$, а

$$
R_{x}^{*}=\sum_{\lambda_{n}>x}\left|a_{n} b_{n}\right| e^{\lambda_{n} \sigma} .
$$

Положим $x_{j}=\inf \left\{x: x \in A_{j}\right\}$, где $A_{j}=\left\{x: x \geqslant \tau_{j}, \ln \mu\left(\tau_{j}\right) \leqslant 2 \ln \mu^{*}(x)\right\} \quad(j \geqslant 1)$. Если $\tau_{j} \notin e_{1}$ при $j \geqslant j_{1}$, то из $(23)$ заключаем, что $x_{j}=\tau_{j}$ при $j \geqslant j_{2}$. В противном случае $\tau_{j} \leqslant x_{j}$. Но поскольку $m\left(e_{1} \cap\left[\tau_{j}, 0\right)\right)=o\left(\left|\tau_{j}\right|\right)$ при $\tau_{j} \rightarrow 0-$, то

$$
\frac{x_{j}-\tau_{j}}{\left|\tau_{j}\right|}=1-\frac{\left|x_{j}\right|}{\left|\tau_{j}\right|}=o(1), \quad \tau_{j} \rightarrow 0-.
$$

Значит, в любом случае $x_{j}=(1+o(1)) \tau_{j}, \tau_{j} \rightarrow 0-$. Следовательно, с учетом (20) получаем, что

$$
\ln \mu^{*}\left(x_{j}\right) \geqslant \frac{1}{2} \ln \mu\left(\tau_{j}\right)>\frac{1}{2} P \tau \Phi_{2}\left(\left|x_{j}\right|^{-1}\right) \quad\left(j \geqslant j_{2}\right),
$$

где $\Phi_{2}(t)=\Phi\left(q^{*} t\right)\left(0<q^{*}<q\right)$. Поскольку $\Phi_{2} \in H$, то опять, применяя леммы 1,2 , при $\sigma \rightarrow 0-$ вне некоторого множества $e_{2} \subset[-1,0), m\left(e_{2} \cap\left[x_{j}, 0\right)\right)=o\left(\left|x_{j}\right|\right), x_{j} \rightarrow 0-$, имеем

$$
R_{x}^{*} \leqslant C \mu^{*}(\sigma)^{-2(1+o(1))}, \quad C=\sum_{n=1}^{\infty} \frac{1}{\lambda_{n}^{m}}
$$


Оценки величин $R_{x}^{*}$ и $m\left(e_{2} \cap\left[x_{j}, 0\right)\right)$ получаются из тех же соображений, что и оценки для величин $R_{v}$ и $m\left(e_{1} \cap\left[\tau_{j}, 0\right)\right)$. Но так как $x_{j}=(1+o(1)) \tau_{j}$ при $\tau_{j} \rightarrow 0-$, то

$$
\frac{m\left(e_{2} \cap\left[\tau_{j}, 0\right)\right)}{\left|\tau_{j}\right|}=o(1), \quad \tau_{j} \rightarrow 0-
$$

Далее, из $(25)$ получаем, что $\lambda_{k(\sigma)} \leqslant x(\sigma)$ при $\sigma_{3} \leqslant \sigma<0, \sigma \notin e_{2}$. Следовательно, из (24) получаем, что при $\sigma \rightarrow 0-$ вне $e_{2}$ выполняется неравенство

$$
\mu^{*}(\sigma) \leqslant \mu(\sigma) \mu^{*}(\sigma)^{o(1)}
$$

т.е.

$$
(1+o(1)) \ln \mu^{*}(\sigma) \leqslant \ln \mu(\sigma) .
$$

Теперь из оценок $(23),(27)$ окончательно получаем, что при $\sigma \rightarrow 0-$ вне множества $e=e_{1} \cup e_{2}$ имеет место асимптотическое равенство

$$
\ln \mu(\sigma)=(1+o(1)) \ln \mu^{*}(\sigma) .
$$

С учетом (22), (26) осталось только заметить, что

$$
\lim _{\tau_{j} \rightarrow 0-} \frac{m\left(e \cap\left[\tau_{j}, 0\right)\right)}{\left|\tau_{j}\right|}=0 .
$$

Таким образом, de $=0$ и достаточность доказана.

Необходимость. Пусть $\Phi$ - некоторая функция из класса $N$, а $\varphi$ - обратная к ней функция. Покажем, что если для любой функции $F \in \bar{D}_{0}(\Lambda, \Phi)$ при $\sigma \rightarrow 0$ - вне некоторого множества $e \subset[-1,0), d e=0$, выполняется равенство (8), то существует функция $w \in W_{\varphi}$ такая, что

$$
\left|b_{n}\right|+\frac{1}{\left|b_{n}\right|} \leqslant e^{w\left(\lambda_{n}\right)} \quad(n \geqslant N) .
$$

Для этого заметим, что условие (28) равносильно предложению: существует функция $g \in W_{\varphi}$ такая, что при $n \geqslant N$

1) $\left|b_{n}\right| \leqslant e^{g\left(\lambda_{n}\right)}$

2) $\left|b_{n}\right|^{-1} \leqslant e^{g\left(\lambda_{n}\right)}$.

Действительно, то, что 1), 2) следуют из (28), очевидно. Обратно, из 1), 2) имеем

$$
\left|b_{n}\right|+\left|b_{n}\right|^{-1} \leqslant 2 e^{g\left(\lambda_{n}\right)} \quad(n \geqslant N) .
$$

Следовательно, $(28)$ имеет место с мажорантой $w(x)=\ln 2+g(x)$.

Предположим, что условие (28) не выполнено. Тогда хотя бы для одной из последовательностей

$$
\left\{\ln \left|b_{n}\right|\right\}_{n=N}^{\infty}, \quad\left\{-\ln \left|b_{n}\right|\right\}_{n=N}^{\infty}
$$

не существует мажоранты вида $w\left(\lambda_{n}\right)$, где $w=W_{\varphi}$. Пусть, ради определенности, последовательность $\left\{\ln \left|b_{n}\right|\right\}_{n=N}^{\infty}$ обладает этим свойством. Это означает, что

$$
\varlimsup_{t \rightarrow \infty} \varphi(t) \int_{t}^{\infty} \frac{\alpha(x)}{x^{2}} d x>0,
$$


где $\alpha=\alpha(t)$ - наименьшая неубьвающая мажоранта последовательности $\left\{\ln \left|b_{n}\right|\right\}_{n=N}^{\infty}$, т.е. $\alpha(t)=\max _{\lambda_{n} \leqslant t}\left\{\ln \left|b_{n}\right|: n \geqslant N\right\}$. Заметим, что $\alpha(t)$ - ступенчатая функция, непрерьвная справа. Не теряя общности, можно считать, что $\alpha(t)>0$ при $n \geqslant N$.

Рассмотрим два случая. Пусть сначала

$$
\lim _{n \rightarrow \infty} \frac{\alpha\left(\lambda_{n}\right) \varphi\left(\lambda_{n}\right)}{\lambda_{n}}=0 .
$$

Если $T=\left\{t_{n}\right\}_{n=1}^{\infty}-$ последовательность всех точек разрыва функции $\alpha(t)$, то, очевидно, $T \subset \Lambda$, т.е. $t_{n}=\lambda_{j_{n}}(n \geqslant 1)$. Пусть $q(0<q<1)$ - произвольное, но фиксированное число, $\beta(t)=q \alpha(t), I_{n}=J\left(t_{n} ; \beta\right), G_{n}=-t_{n} I_{n} \quad(n \geqslant 1)$. Положим

$$
a_{k}= \begin{cases}e^{-G_{1}}, & \text { если } k=1,2, \ldots, j_{1} ; \\ e^{-G_{n}}, & \text { если } k=j_{n}(n \geqslant 1) \\ e^{-\gamma_{n}\left(\lambda_{k}\right)-1}, & \text { если } j_{n}<k<j_{n+1}(n \geqslant 1),\end{cases}
$$

где $y=\gamma_{n}(x)$ - уравнение прямой, проходящей через точки $P_{n}=\left(t_{n}, G_{n}\right)$ и $P_{n+1}=$ $\left(t_{n+1}, G_{n+1}\right)$.

Убедимся, что $R_{n} \uparrow 0$ при $n \rightarrow \infty$, где

$$
R_{n}=\frac{G_{n+1}-G_{n}}{t_{n+1}-t_{n}}
$$

Действительно, $R_{n}=-I_{n}+\beta\left(t_{n}\right) / t_{n}(n \geqslant 1)$ (мы воспользовались тем, что $\beta(t)=q \alpha(t)$, а $\alpha(t)=\alpha\left(t_{n}\right)$ при $\left.t_{n} \leqslant t<t_{n+1}\right)$. Отсюда получаем, что

$$
R_{n+1}-R_{n}=q\left(\frac{\alpha\left(t_{n+1}\right)-\alpha\left(t_{n}\right)}{t_{n+1}}\right)>0 \quad(n \geqslant 1) .
$$

Но так как $G_{n}=o\left(t_{n}\right)$ при $n \rightarrow \infty$, то, действительно, $R_{n} \uparrow 0$ при $n \rightarrow \infty$. Следовательно, совокупность всех отрезков прямых $y=\gamma_{n}(x)$, соединяющих точки $P_{n}$ и $P_{n+1}$ $(n \geqslant 1)$, есть вьпуклый полигон Ньютона $L(F)$ для ряда Дирихле

$$
F(s)=\sum_{k=1}^{\infty} a_{k} e^{\lambda_{k} s}
$$

а поскольку точки $\left(\lambda_{k},-\ln \left|a_{k}\right|\right)$ при $j_{n}<k<j_{n+1}(n \geqslant 1)$ лежат вьше $L(F)$, то вершинами полигона $L(F)$ являются как раз точки

$$
P_{n}=\left(t_{n}, G_{n}\right), \quad t_{n}=\lambda_{j_{n}} \quad(n \geqslant 1) .
$$

Оценим максимальньй член $\mu(\sigma)$ ряда (31) сверху. Для $R_{n-1} \leqslant \sigma<R_{n}$ имеем

$$
\begin{aligned}
\ln \mu(\sigma) & =-G_{n}+t_{n} \sigma=t_{n}\left(I_{n}+\sigma\right) \\
& <\frac{t_{n} t_{n+1}}{t_{n+1}-t_{n}} \int_{t_{n}}^{t_{n+1}} \frac{\beta(x)}{x^{2}} d x=q \alpha\left(t_{n}\right) \quad(n \geqslant 1) .
\end{aligned}
$$


С другой стороны,

$$
\mu^{*}(\sigma) \geqslant\left|a_{j_{n}} b_{j_{n}}\right| e^{\lambda_{j_{n}} \sigma}, \quad \lambda_{j_{n}}=t_{n} \quad(n \geqslant 1)
$$

Следовательно, для $R_{n-1} \leqslant \sigma<R_{n}$ получаем, что

$$
\ln \mu^{*}(\sigma) \geqslant \alpha\left(t_{n}\right)+t_{n}\left(I_{n}+\sigma\right)=\alpha\left(t_{n}\right)+\ln \mu(\sigma)>\alpha\left(t_{n}\right) \quad(n \geqslant 1) .
$$

Но тогда из $(32),(33)$ окончательно получаем, что для $R_{n-1} \leqslant \sigma<R_{n}$ выполняется неравенство

$$
\ln \mu(\sigma)<q \ln \mu^{*}(\sigma) \text {. }
$$

Значит,

$$
\varlimsup_{\sigma \rightarrow 0-} \frac{\ln \mu(\sigma)}{\ln \mu^{*}(\sigma)} \leqslant q<1
$$

и оценка (8) для построенного нами ряда (31) не имеет места. Осталось только показать, что $F \in \bar{D}_{0}(\Lambda, \Phi)$.

Поскольку $\Phi \in N$, то $(\varphi(t) / t) \downarrow 0$ при $t \uparrow \infty$. Следовательно, учитывая (29) и применяя лемму 3 , получаем, что

$$
\varphi\left(\tau_{k}\right) J\left(\tau_{k} ; \alpha\right) \geqslant \tau>0
$$

где $\left\{\tau_{k}\right\}_{k=1}^{\infty}-$ некоторая подпоследовательность последовательности $T=\left\{t_{n}\right\}$ всех точек разрьва функции $\alpha(t)$.

Пусть $\tau_{k}=t_{n_{k}}(k \geqslant 1)$ и $R_{n_{k}-1} \leqslant \sigma<R_{n_{k}}$. Тогда $\lambda_{\nu(\sigma)}=\tau_{k},\left|R_{n_{k}}\right|<|\sigma| \leqslant\left|R_{n_{k}-1}\right|$ $(\nu(\sigma)$ - центральньй индекс ряда $(31))$.

Учитывая (30), (34), а также равенство $R_{n_{k}}=-I_{n_{k}}+q \alpha\left(t_{n_{k}}\right) / t_{n_{k}}(k \geqslant 1)$, имеем

$$
\varphi\left(\tau_{k}\right)\left|R_{n_{k}}\right|=\varphi\left(\tau_{k}\right) I_{n_{k}}-q \frac{\alpha\left(\tau_{k}\right) \varphi\left(\tau_{k}\right)}{\tau_{k}} \geqslant \frac{\tau}{2}>0 \quad\left(k \geqslant k_{0}\right) .
$$

Следовательно, при $k \geqslant k_{0}$

$$
\varphi\left(\lambda_{\nu(\sigma)}\right) \geqslant \frac{\tau q}{2|\sigma|}, \quad\left|R_{n_{k}}\right|<|\sigma| \leqslant\left|R_{n_{k}-1}\right| \quad(0<q<1) .
$$

Отсюда при $k \geqslant k_{0},\left|R_{n_{k}}\right|<|\sigma| \leqslant\left|R_{n_{k}-1}\right|$, имеем

$$
\lambda_{\nu(\sigma)} \geqslant \Phi\left(\frac{\tau q}{2|\sigma|}\right)
$$

Далее, из известного представления [1]

$$
\ln \mu(\sigma)=\ln \mu(-1)+\int_{-1}^{\sigma} \lambda_{\nu(t)} d t
$$

получаем, что

$$
\ln \mu\left(\frac{\sigma}{2}\right) \geqslant \int_{\sigma}^{\sigma / 2} \lambda_{\nu(t)} d t \geqslant \frac{|\sigma|}{2} \lambda_{\nu(\sigma)}
$$


Следовательно, учитьвая (35), имеем

$$
\ln \mu\left(\frac{\sigma}{2}\right) \geqslant \frac{|\sigma|}{2} \Phi\left(\frac{\tau q}{2|\sigma|}\right), \quad\left|R_{n_{k}}\right|<|\sigma| \leqslant\left|R_{n_{k}-1}\right| \quad\left(k \geqslant k_{0}\right) .
$$

Таким образом,

$$
\varlimsup_{\sigma \rightarrow 0-} \frac{\ln \mu(\sigma)}{|\sigma| \Phi(a /|\sigma|)} \geqslant 1, \quad a=\frac{\tau q}{2} .
$$

Значит, $F \in \bar{D}_{0}(\Lambda, \Phi)$ и в случае вьполнения условия (30) пример построен.

Пусть теперь

$$
\varlimsup_{n \rightarrow \infty} \frac{\varphi\left(\lambda_{n}\right) \alpha\left(\lambda_{n}\right)}{\lambda_{n}}>0 .
$$

Тогда существует подпоследовательность $\left\{\lambda_{n_{k}}\right\}$ такая, что

$$
\alpha\left(\lambda_{n_{k}}\right) \geqslant \beta \frac{\lambda_{n_{k}}}{\varphi\left(\lambda_{n_{k}}\right)} \quad(k \geqslant 1, \beta>0) .
$$

Рассмотрим ряд Дирихле

$$
F(s)=\sum_{n=1}^{\infty} a_{n} e^{\lambda_{n} s} \quad(s=\sigma+i t)
$$

где

$$
a_{n}= \begin{cases}\exp \left(\frac{\beta}{2} \frac{\lambda_{n}}{\varphi\left(\lambda_{n}\right)}\right), & \text { если } n=n_{k}, \\ 0, & \text { если } n \neq n_{k} .\end{cases}
$$

Пусть $\nu=\nu(\sigma)$ - центральньй индекс ряда (37). Тогда

$$
\ln \mu(\sigma)=\max _{k \geqslant 1}\left(\frac{\beta}{2} \frac{\lambda_{n_{k}}}{\varphi\left(\lambda_{n_{k}}\right)}-\lambda_{n_{k}}|\sigma|\right)=\frac{\beta}{2} \frac{\lambda_{\nu}}{\varphi\left(\lambda_{\nu}\right)}-\lambda_{\nu}|\sigma|<\frac{\beta}{2} \frac{\lambda_{\nu}}{\varphi\left(\lambda_{\nu}\right)},
$$

$\ln \mu^{*}(\sigma) \geqslant \ln \left|a_{\nu}\right|+\ln \left|b_{\nu}\right|-\lambda_{\nu}|\sigma|=\ln \mu(\sigma)+\ln \left|b_{\nu}\right|>\ln \left|b_{\nu}\right|=\alpha\left(\lambda_{\nu}\right) \geqslant \beta \frac{\lambda_{\nu}}{\varphi\left(\lambda_{\nu}\right)}$.

Следовательно,

$$
\varlimsup_{\sigma \rightarrow 0-} \frac{\ln \mu(\sigma)}{\ln \mu^{*}(\sigma)} \leqslant \frac{1}{2} .
$$

Убедимся, что $F \in \bar{D}_{0}(\Lambda, \Phi)$. Действительно, для любого $\sigma<0$

$$
\ln \mu(\sigma) \geqslant \frac{\beta}{2} \frac{m_{k}}{\varphi\left(m_{k}\right)}-m_{k}|\sigma| \quad\left(m_{k}=\lambda_{n_{k}}, \quad k \geqslant 1\right) .
$$

Пусть $\sigma=\sigma_{k}-$ корень уравнения

$$
m_{k}=\Phi\left(\frac{\beta}{4|\sigma|}\right) \quad\left(k \geqslant k_{0}\right) .
$$

Тогда

$$
\ln \mu\left(\sigma_{k}\right) \geqslant \Phi\left(\frac{\beta}{4\left|\sigma_{k}\right|}\right)\left|\sigma_{k}\right| \quad\left(k \geqslant k_{0}\right) .
$$

Значит,

$$
\varlimsup_{\sigma \rightarrow 0-} \frac{\ln \mu(\sigma)}{|\sigma| \Phi(a /|\sigma|)} \geqslant 1 \quad\left(a=\frac{\beta}{4}\right) .
$$

Таким образом, $F \in \bar{D}_{0}(\Lambda, \Phi)$ и необходимость полностью доказана. 
3. Некоторые применения к рядам Дирихле заданного роста. Пусть функция $F$ принадлежит $D_{0}(\Lambda)$ и максимальньй член представляющего ее ряда $(2)$ имеет ненулевой порядок по Ритту в полуплоскости $\Pi_{0}$, т.е. [8]

$$
\varlimsup_{\sigma \rightarrow 0-} \frac{\ln \ln \mu(\sigma)}{|\sigma|^{-1}}=\rho_{R}>0 \text {. }
$$

Тогда существует последовательность $\left\{\sigma_{k}\right\}, \sigma_{k} \uparrow 0$, такая, что для $\sigma=\sigma_{k}$

$$
\ln \mu(\sigma)>|\sigma| e^{a|\sigma|^{-1}} \quad\left(0<a<\rho_{R}\right)
$$

Отсюда

$$
\varlimsup_{\sigma \rightarrow 0-} \frac{\ln \mu(\sigma)}{|\sigma| \Phi\left(a|\sigma|^{-1}\right)}>0
$$

где $\Phi(t)=e^{t}$. Ясно, что $\Phi \in N, \varphi(t)=\ln t$. Имея это в виду, через $\bar{D}_{0}(\Lambda, R)$ обозначим подкласс функций из $\bar{D}_{0}(\Lambda, \Phi)$, для которых выполняется условие $(38)$.

Имеет место следующая

Tеорема D. Для того чтобы для любой функиии $F \in \bar{D}_{0}(\Lambda, R)$ при $\sigma \rightarrow 0-$ вне некоторого множества е $\subset[-1,0)$ нулевой нижней плотности выполнялось асимптотическое равенство

$$
\ln \mu(\sigma)=(1+o(1)) \ln \mu^{*}(\sigma)
$$

необходимо и достаточно, чтобы

$$
\lim _{t \rightarrow \infty} \ln t \int_{t}^{\infty} \frac{\alpha(x)}{x^{2}} d x=0 .
$$

Здесь $\alpha=\alpha(t)$ - наименьшая неубывающая махсоранта последовательнос$m u\left\{\left|b_{n}\right|+\left|b_{n}\right|^{-1}\right\}_{n=N}^{\infty}$.

Теорема D есть простое следствие теоремы C.

Легко сфформулировать соответствующую теорему и в случае, когда $\Phi(t)=\exp _{k} t$, где

$$
\exp _{k} t=\underbrace{\exp \exp \cdots \exp }_{k \text { раз }} t
$$

Мы ограничимся формулировкой теоремы только о степенных рядах, непосредственно вытекающей из теоремы D.

Пусть область сходимости степенного ряда

$$
f(z)=\sum_{n=0}^{\infty} a_{n} z^{n}
$$

- единичный круг $D(0,1)=\{z:|z|<1\}$. Для функции $\Phi(t)=e^{t}$ класс $\bar{K}(\Phi)$ представляет собой множество

$$
\bar{K}(\exp )=\left\{f: \sup _{\tau>0} \varlimsup_{r \rightarrow 1-} \frac{\ln \mu_{f}(r)}{(1-r) \exp \left[\tau(1-r)^{-1}\right]}>0\right\},
$$

где $\mu_{f}(r)$ - максимальньй член ряда (40).

Справедлива следующая 
Tеорема Е. Для того чтобъ для любой функиии $f \in \bar{K}(\exp )$ при $r \rightarrow 1-$ вне некоторого множества $e_{1} \subset[0,1)$ такого, что $d^{\prime} e_{1}=0$, имело место асимптотическое равенство

$$
\ln \mu_{f}(r)=(1+o(1)) \ln \mu_{f}^{*}(r),
$$

необходимо и достаточно, чтобы выполнялось условие (39).

Здесь

$$
d^{\prime} e_{1}=\varliminf_{r \rightarrow 1-} \frac{m\left(e_{1} \cap[r, 1)\right)}{1-r} .
$$

\section{СПИСОК ЦИТИРОВАННОЙ ЛИТЕРАТУРЫ}

[1] Леонтьев А. Ф. Ряды экспонент. М.: Наука, 1976.

[2] Гайсин А. М. Оценка ряда Дирихле с лакунами Фейера на кривых // Докл. РАН. 2000. T. 370. №6. C. 735-737.

[3] Wiman A. Über den Zusammenhang zwischen dem Maximalbetrage einer analytischen Funktion und dem grössten Betrage bei gegebenem Argumente der Function // Acta Math. 1916/18. V. 41. P. 1-28.

[4] Скаскив О.Б. К теореме Вимана о минимуме модуля аналитической в единичном круге функции // Изв. АН СССР. Сер. матем. 1989. Т. 53. № 4. С. 833-850.

[5] Гайсин А. М. Поведение логарифма модуля ряда Дирихле, сходящегося в полуплоскости // Изв. РАН. Сер. матем. 1994. Т. 58. № 4. С. 173-185.

[6] Красносельский М.А., Рутицкий Я. Б. Вьпуклые функции и пространства Орлича. М.: Физматгиз, 1958.

[7] Цегелик Г. Г. Свойства мажоранты и диаграммы Ньютона функции, аналитической в круге // Укр. матем. ж. 1977. Т. 29. № 4. С. 560-562.

[8] Гайсин А. М. Оценка роста функции, представленной рядом Дирихле, в полуполосе // Матем. сб. 1982. Т. 117 (159). № 3. С. 412-424. 